\title{
AN INEQUALITY FOR THE COUPLING MOMENT IN THE CASE OF TWO INHOMOGENEOUS MARKOV CHAINS
}

UDC 519.21

\author{
V. V. GOLOMOZY $\breve{I}$
}

\begin{abstract}
We consider discrete Markov chains with phase space $\{0,1, \ldots\}$ and study conditions under which the expectation of the first coupling moment for two independent discrete time inhomogeneous Markov chains is finite. The coupling moment is defined as the first time when both chains simultaneously visit the zero state. Some special cases are considered where a bound for the expectation of the coupling moment is available.
\end{abstract}

\section{INTRODUCTION}

Some applications of the results obtained in [27] are considered in the current paper. We present some corollaries to Theorem 5.1 of [27] that allow one to obtain sufficient conditions for the existence of the expectation of the coupling moment in the case of two time inhomogeneous Markov chains. With the help of these general results, we obtain a number of applications for specific Markov chains.

A stronger result is presented in the current paper in the case of homogeneous Markov chains (see Theorem 4.4 below). This result weakens to some extent the condition of the strict aperiodicity, $g_{1}^{1}+g_{1}^{2}>0$, used in [31] to prove a similar result. In the current paper, we show that the constant involved in the bound for the coupling moment can easily be evaluated in the case of homogeneous renewal processes if the second moments exist and if a weaker condition for the aperiodicity is assumed instead of the condition $g_{1}^{1}+g_{1}^{2}>0$.

\section{Sufficient COnditions For the EXISTEnCE OF THE EXPECTATION OF THE COUPLING MOMENT}

We start with a result proved in [27] (Theorem 5.1 therein). For convenience, we provide its statement below as well as necessary notation and definitions.

Consider two time inhomogeneous Markov chains $\left(X_{t}^{1}, t \geq 0\right)$ and $\left(X_{t}^{2}, t \geq 0\right)$ assuming values in the space $E=\{0,1, \ldots\}$. The chains are defined by their transient probabilities at step $s$, namely $P_{s}(x, A, 1)$ and $P_{s}(x, A, 2)$ for the chains $X_{t}^{1}$ and $X_{t}^{2}$, respectively. Then one can evaluate the transient probabilities over $n>0$ steps:

$$
P^{t, n}(x, A, l)=\left(\prod_{k=0}^{n-1} P_{t+k}\right)(x, A, l) .
$$

Using this family of transient probabilities and the initial distributions $\mu^{l}(\cdot)$ one can construct the probability space $(\Omega, \mathcal{F}, \mathrm{P})$ where independent chains $\left(X_{t}^{l}\right), l \in\{0,1\}$, leave.

2010 Mathematics Subject Classification. Primary 60J45; Secondary 60A05, 60K05.

Key words and phrases. Coupling theory, coupling method, maximal coupling, discrete Markov chains, stability of distributions. 
Moreover,

$$
\mathrm{P}\left\{X_{s}^{l} \in A\right\}=\int_{E} \mu^{l}(d x) P^{0, s}(x, A, l), \quad \mathrm{P}\left\{X_{s+1}^{l} \in A \mid X_{s}^{l}=x\right\}=P_{s}(x, A, l) .
$$

Let $\left(\theta_{k}^{l}\right), l \in\{0,1\}$, be the sequences of renewal intervals:

$$
\theta_{0}^{l}=\inf \left\{t \geq 0: X_{t}=0\right\}, \quad \theta_{m}^{l}=\inf \left\{t>\theta_{m-1}: X_{t}=0\right\}, \quad m>1,
$$

defined in the joint probability space $(\Omega, \mathcal{F}, \mathrm{P})$. The classes of random variables $\left\{\theta_{k}^{1}\right\}_{k \geq 0}$ and $\left\{\theta_{k}^{2}\right\}_{k \geq 0}$ are independent. The random variable $\theta_{k}^{l}$ with $l=1,2$ and $k>0$ assumes positive integer values, while $\theta_{0}^{l}$ assumes nonnegative integer values.

The renewal sequences are defined as

$$
\tau_{n}^{l}=\sum_{k=0}^{n} \theta_{k}^{l}, \quad l=1,2 .
$$

We assume that every two subsequent random variables in each class are conditionally independent for all fixed $\tau$. This means that, for all $k, t$, and $l$,

$$
\mathrm{E}\left[f\left(\theta_{k}^{l}\right) g\left(\theta_{k+1}^{l}\right) \mid \tau_{k}^{l}\right]=\mathrm{E}\left[f\left(\theta_{k}^{l}\right) \mid \tau_{k}^{l}\right] \mathrm{E}\left[g\left(\theta_{k+1}^{l}\right) \mid \tau_{k}^{l}\right]
$$

for all bounded Borel functions $f$ and $g$. A motivation for this assumption can be found in the paper [27].

Let

$$
g_{n}^{t, l}=\mathrm{P}\left\{\theta_{k}^{l}=n \mid \tau_{k-1}=t\right\}, \quad l=1,2, n \geq 0,
$$

be the conditional distributions of the random variables $\theta_{k}^{l}$. Note that these distributions do not depend on $k$. We also assume that $g_{0}^{t, l}=\mathrm{P}\left\{\theta_{k}^{l}=0 \mid \tau_{k-1}=t\right\}=0$. The random variables $\theta_{k}^{l}, k \geq 1$, are treated as a renewal step, while $\theta_{0}^{l}$ is treated as a delay.

We say $T>0$ is a coupling moment if

$$
T=\min \left\{t>0: \exists m, n: t=\tau_{m}^{1}=\tau_{n}^{2}\right\} .
$$

Our aim is to find conditions under which $T<\infty$ almost surely or $\mathrm{E}[T]<\infty$.

By $u_{n}^{(t, l)}$ we denote the renewal sequence constructed from the process $\tau^{l}$. In other words, $u_{n}^{(t, l)}$ is the probability that a renewal occurs at the moment $t+n$ given that a renewal occurred at the moment $t$. Formally, $u_{n}^{(t, l)}$ is defined as

$$
u_{0}^{(t, l)}=1, \quad u_{n}^{(t, l)}=\sum_{k=0}^{n} u_{k}^{(t, l)} g_{n-k}^{t+k, l} .
$$

\section{Definition of RAndom variables $\theta^{l}(t)$}

As we have seen above, the distribution of the $(k+1)^{\text {th }}$ renewal interval is completely determined by the random variable $\tau_{k}$, that is, by the moment when the preceding renewal occurs. Recall that this distribution does not depend on the index $k$. This explains our notation $g_{n}^{t, l}$ and $u_{n}^{(t, l)}$. Our aim is to construct random variables $\theta^{l}(t)$ in such a way that $g_{n}^{t, l}$ is the distribution of $\theta^{l}(t)$.

Throughout this section we drop the superscript $l$. Assume that $X_{t}$ is a time inhomogeneous Markov chain whose transient probabilities at step $t$ are $P_{t}(x, A)$. As above, let

$$
P^{t, n}(x, A)=\left(\prod_{k=0}^{n-1} P_{t+k}\right)(x, A)
$$

be the transient probability over the time from $t$ to $t+n$. 
For every $t$, we define a probability space $\left(\Omega_{t}, \mathcal{F}_{t}, \mathrm{P}_{t}\right)$ as the canonical space for the Markov chain $X_{t+n}$ that starts from the origin (in other words, the initial measure is concentrated at zero). Put

$$
\theta(t)=\min \left\{j>0: X_{t+j}=0\right\} .
$$

Let $g_{n}^{t}=\mathrm{P}_{t}\{\theta(t)=n\}$ be the distribution of the random variable $\theta(t)$. Then

$$
g_{n}^{t}=\int_{(E \backslash\{0\})^{n-1}} P_{t}\left(0, d x_{0}\right) P_{t+1}\left(x_{0}, d x_{1}\right) \cdots P_{t+n-1}\left(x_{n-1},\{0\}\right) .
$$

As above, let $\theta^{l}(t)$ be the first time when the chain $\left(X_{t+k}^{l}, k \geq 0\right)$ visits zero if it starts from the origin. Then the distribution of the random variable $\theta^{l}(t)$ is $\left(g_{n}^{t, l}\right)_{n \geq 0}$.

Let

$$
D_{n}(t)=\min \left\{j \geq 0: X_{t+n+j}=0\right\} .
$$

The random variable $D_{n}(t)$ means the time left until the chain reaches $\{0\}$ after the moment $t+n$ given $X_{t}=0$. Note that $D_{n}(t)$ and $\theta(t)$ are defined in a common probability space $\left(\Omega_{t}, \mathcal{F}_{t}, \mathrm{P}_{t}\right)$.

The following assertion plays a crucial role in the proof of the main result of this paper.

Lemma 3.1. If the family of distributions $g_{n}^{t}$ (or random variables $\theta(t)$ ) is uniformly integrable, then, for an arbitrary $\rho \in(0,1)$, there exists a constant $C=C(\rho) \geq 0$ such that

$$
\mathrm{E}_{t}\left[D_{n}(t)\right] \leq \rho n+C
$$

for all $t$.

Theorem 3.1. Assume that

(1) the family of random variables $\theta^{l}(t)$ is uniformly integrable (in other words, the family of probability measures $g_{n}^{t, l}$ is uniformly integrable);

(2) there exist a constant $\gamma>0$ and a positive integer number $n_{0}>0$ such that $u_{n}^{(t, l)} \geq \gamma$ for all $t, l$, and $n \geq n_{0}$.

Then the expectation of the coupling moment exists:

$$
\mathrm{E}[T]<\infty .
$$

\section{Corollaries of Theorem 3.1}

Corollary 4.1. Let $X_{t}$ and $X_{t}^{\prime}$ be two homogeneous aperiodic Markov chains such that

$$
\max \left\{\mathrm{E}\left[\theta_{0}\right], \mathrm{E}\left[\theta_{0}^{\prime}\right], \mathrm{E}\left[\theta_{1}\right], \mathrm{E}\left[\theta_{1}^{\prime}\right]\right\}<\infty .
$$

Then $\mathrm{E}[T]<\infty$.

Proof. Since the distributions of all $\theta_{k}, k \geq 1$, are identical and the same is true for $\theta_{k}^{\prime}$, $k \geq 1$, we conclude that there are at most four different distributions in the family $g_{n}^{t, l}$ and thus this family is uniformly integrable.

Since the chains are nonperiodic and have finite return moments, the renewal theorem implies that $u_{n} \rightarrow 1 / \mathrm{E}\left[\theta_{1}\right]>0$ and $u_{n}^{\prime} \rightarrow 1 / \mathrm{E}\left[\theta_{1}^{\prime}\right]>0$ as $n \rightarrow \infty$. Thus both sequences are separated from zero starting with some number.

Therefore all assumptions of Theorem 3.1 hold.

Remark 4.1. Verifying assumptions of Theorem 3.1 is a complicate technical problem in general. We consider several partial cases where checking these assumptions is rather easy. 
The following results provide sufficient conditions that imply condition (2) of Theorem 3.1. It is worth mentioning that the proof of the next two results follows the idea of the proof of the corresponding assertion in the monograph [3].

Theorem 4.1. Let $\theta_{n}$ be a renewal sequence generated by an inhomogeneous Markov chain and let $\left(g_{n}^{(t)}\right)$ be the family of conditional distributions of $\left(\theta_{n}\right)$. Assume that

1. there exist $m$ positive integer numbers $l_{1}, \ldots, l_{m}$ with $\operatorname{gcd}\left(l_{1}, \ldots, l_{m}\right)=1$ such that

$$
\inf _{t, i} g_{l_{i}}^{(t)}>0
$$

2. the sequence $\theta_{n}$ is stochastically dominated; that is $G_{n}^{(t)} \leq \hat{G}_{n} \rightarrow 0, n \rightarrow \infty$; moreover $\hat{g}_{l_{1}}>0$ and the moment $\hat{\mu}=\sum_{n \geq 0} \hat{G}_{n}$ exists.

Then there exist a number $n_{0}$ and a constant $\gamma>0$ such that $u_{n}^{(t)}>\gamma$ for all $n \geq n_{0}$ and $t>0$.

Theorem 4.2. Using the notation of Theorem 3.1 let

a) there exist $n_{0}$ such that $g_{n}^{(t, l)}>0$ for all $t, l$, and $n \geq n_{0}$;

b) there exist $\gamma_{i}>0, i=0, \ldots, n_{0}-1$, such that $g_{n_{0}+i}^{(t, l)} \geq \gamma_{i}$ for all $t, l$ and every $i=0, \ldots, n_{0}-1$.

Then condition (2) of Theorem 3.1 holds.

Corollary 4.2. Let $\left(\hat{g}_{n}\right)$ be a sequence that stochastically dominates the family of distributions of $\left(g_{n}^{t, l}\right)$; that is, $G_{n}^{t, l} \leq \hat{G}_{n}$. If $\hat{g}_{1}>0$, then condition (2) of Theorem 3.1 holds with $n_{0}=1$.

Theorem 4.3. If there are only a finite number of different distributions in the family $\left\{\left(g_{n}^{t, l}, n \geq 0\right)\right\}$ and each of them is aperiodic, then condition (2) of Theorem 3.1 holds for some $n_{0}$.

Widely known are some general conditions for the uniform integrability of a family of random variables. In particular,

1) the family $\theta_{n}$ is uniformly integrable if and only if $\theta_{n}$ is stochastically dominated by some random variable $\zeta$ with a finite expectation

or

2) if $\sup _{n} \mathrm{E}\left[\theta_{n}^{p}\right]<\infty$ for some $p>1$, then the family $\theta_{n}$ is uniformly integrable.

The method of test functions can also be used to check whether or not moments are finite.

The following result is obtained under much stronger assumptions than those in the preceding theorems. On the other hand, it allows one to estimate the expectation of the coupling moment.

Theorem 4.4. Consider two homogeneous independent aperiodic renewal processes $\theta^{(l)}$, $l \in\{1,2\}$. Assume that

(1) the second moments $\mu_{2}^{(l)}$ are finite;

(2) there exist $\gamma>0$ and $n_{0}$ such that $u_{n}^{(l)} \geq \gamma$ for all $l$ and $n>n_{0}$.

Then

$$
\mathrm{E}[T] \leq \mathrm{E}\left[\max \left(\theta_{0}^{1}, \theta_{0}^{2}\right)\right]+\gamma^{-1} \max \left\{\frac{\mu_{2}^{(1)}}{\mu^{(1)}}, \frac{\mu_{2}^{(2)}}{\mu^{(2)}}\right\}
$$


Remark 4.2. The assumptions of Theorem 4.4 imply conditions of Theorem 3.1. On the other hand, the proof of Theorem 4.4 is essentially different from that of Theorem 3.1 and uses Daley's inequality, valid for the homogeneous case (there is no analogue of Daley's inequality for the inhomogeneous case). Therefore Theorem 4.4 is stated only for homogeneous renewal processes.

\section{Applications for homogeneous Markov Chains}

Inverse renewal chain. First we consider two homogeneous Markov chains defined by their transient matrices:

$$
P=\left(\begin{array}{ccccc}
b_{0} & 1-b_{0} & 0 & 0 & \cdots \\
b_{1} & 0 & 1-b_{1} & 0 & \cdots \\
b_{2} & 0 & 0 & 1-b_{2} & \ldots \\
b_{3} & 0 & 0 & 0 & 1-b_{3} \\
& & \cdots & &
\end{array}\right)
$$

and

$$
Q=\left(\begin{array}{ccccc}
c_{0} & 1-c_{0} & 0 & 0 & \ldots \\
c_{1} & 0 & 1-c_{1} & 0 & \ldots \\
c_{2} & 0 & 0 & 1-c_{2} & \ldots \\
c_{3} & 0 & 0 & 0 & 1-c_{3}
\end{array}\right)
$$

The sequences $G_{n}^{l}$ and $g_{n}^{l}$ are evaluated as follows:

$$
\begin{gathered}
G_{n}^{1}=\prod_{k=0}^{n-1}\left(1-b_{k}\right), \quad G_{n}^{2}=\prod_{k=0}^{n-1}\left(1-c_{k}\right), \quad n \geq 1, \\
g_{1}^{1}=b_{0}, \quad g_{n}^{1}=\prod_{k=0}^{n-2}\left(1-b_{k}\right) b_{n-1}, \quad n \geq 2, \\
g_{1}^{2}=c_{0}, \quad g_{n}^{2}=\prod_{k=0}^{n-2}\left(1-c_{k}\right) c_{n-1}, \quad n \geq 2 .
\end{gathered}
$$

Then $m_{j}^{l}$, the expectation of the time needed to reach the state 0 from a point $j$, is given by

$$
m_{j}^{1}=1+\sum_{k \geq 1} \prod_{i=0}^{k-1}\left(1-b_{j+i}\right), \quad m_{j}^{2}=1+\sum_{k \geq 1} \prod_{i=0}^{k-1}\left(1-c_{j+i}\right) .
$$

The second moment of return to the origin is equal to

$$
m^{1,2}=b_{0}+\sum_{n \geq 2} n^{2} \prod_{k=0}^{n-2}\left(1-b_{k}\right) b_{n-1}, \quad m^{2,2}=c_{0}+\sum_{n \geq 2} n^{2} \prod_{k=0}^{n-2}\left(1-c_{k}\right) c_{n-1} .
$$

Now we are in position to state different sufficient conditions for the existence of the expectation of the coupling moment.

Theorem 5.1. Let independent Markov chains $X_{n}$ and $X_{n}^{\prime}$ with transient probabilities $P$ and $Q$ start from initial states $l$ and $j$, respectively. Assume that

(1) $b_{n}>0$ and $c_{n}>0, n \geq 1$;

(2) $\max \left\{m_{l}^{1}, m_{j}^{2}, m_{0}^{l}\right\}<\infty$.

Then the expectation of the moment of a simultaneous visit of the origin by the chains $X$ and $X^{\prime}$ is finite; that is, $\mathrm{E}[T]<\infty$. 
Proof. Assumption (1) implies that the chains $X$ and $X^{\prime}$ are aperiodic. The finiteness of the moments $\theta_{j}^{l}, j \geq 0$, follows from condition (2). therefore the corollary to Theorem 3.1 yields the finiteness of the expectation of the coupling moment.

Remark 5.1. Theorem 5.1 does not require any of the assumptions $b_{0}>0$ or $g_{1}^{l}>0$.

If one adds the assumption that second moments exist to the set of assumptions of Theorem 5.1, then one can get a bound for the expectation of the coupling moment.

Theorem 5.2. Let independent Markov chains $X_{n}$ and $X_{n}^{\prime}$ with transient probabilities $P$ and $Q$ start from the initial states $l$ and $j$, respectively. Assume that

(1) $b_{n}>0$ and $c_{n}>0, n \geq 1$;

(2) $\max \left\{m_{l}^{1}, m_{j}^{2}, m^{l, 2}\right\}<\infty$.

Then

where

$$
\mathrm{E}[T]<m_{l}^{1}+m_{j}^{2}+\gamma^{-1} \max \left\{\frac{m^{1,2}}{m_{0}^{1}}, \frac{m^{2,2}}{m_{0}^{2}}\right\},
$$

$$
\begin{gathered}
\gamma=\gamma_{0}\left(1-\hat{G}_{1}\right)^{\left(\max \left\{m_{0}^{1}, m_{0}^{2}\right\}-\hat{G}_{1}\right) / \hat{G}_{1}}, \\
\hat{G}_{1}=\max \left\{G_{1}^{1}, G_{1}^{2}\right\}, \\
\gamma_{0}=\min \left\{\left(1-b_{0}\right) b_{1},\left(1-b_{0}\right)\left(1-b_{1}\right) b_{2},\left(1-c_{0}\right) c_{1},\left(1-c_{0}\right)\left(1-c_{1}\right) c_{2}\right\} .
\end{gathered}
$$

Proof. We apply Theorem 4.4. First we note that

$$
\mathrm{E}\left[\max \left\{\theta_{0}^{1}, \theta_{0}^{2}\right\}\right] \leq \mathrm{E}\left[\theta_{0}^{1}\right]+\mathrm{E}\left[\theta_{0}^{2}\right]=m_{l}^{1}+m_{j}^{2} .
$$

To complete the proof, one needs to check condition (2) of Theorem 4.4. We use Theorem 4.2 for this purpose. We are going to show that $\min \left\{g_{2}^{l}, g_{3}^{l}\right\}>0$. Indeed,

$g_{2}^{1}=\left(1-b_{0}\right) b_{1}, \quad g_{3}^{1}=\left(1-b_{0}\right)\left(1-b_{1}\right) b_{2}, \quad g_{2}^{2}=\left(1-c_{0}\right) c_{1}, \quad g_{3}^{3}=\left(1-c_{0}\right)\left(1-c_{1}\right) c_{2}$.

Then assumption (1) of Theorem 5.2 implies that $\gamma_{0}=\min \left\{g_{2}^{l}, g_{3}^{l}\right\}>0$. The expression for $\gamma$ is derived from Theorem 4.2 .

Remark 5.2. The bounds for moments become simpler if there exists $\gamma>0$ such that $b_{n}>\gamma$ and $c_{n}>\gamma$. In this case, however, one can apply a stronger result obtained in 28 .

Remark 5.3. A result of [31, similar to Theorem 4.4, relies on the condition $g_{1}^{1}+g_{1}^{2}>0$. Theorem 4.4 uses a weaker assumption than that in 31] under which both $g_{1}^{1}$ and $g_{1}^{2}$ may equal zero. An example of such a case is presented in Theorem 5.2. It is worth mentioning that bounds of the coupling moment obtained in [31] and in Theorem 4.4 are very similar.

Random walk. Consider two homogeneous Markov chains defined by the following transient matrices:

and

$$
P=\left(\begin{array}{ccccc}
p & 1-p & 0 & 0 & \cdots \\
p & 0 & 1-p & 0 & \cdots \\
0 & p & 0 & 1-p & \ldots \\
0 & 0 & p & 0 & 1-p \\
& & \cdots & &
\end{array}\right)
$$

$$
Q=\left(\begin{array}{ccccc}
q & 1-q & 0 & 0 & \cdots \\
q & 0 & 1-q & 0 & \cdots \\
0 & q & 0 & 1-q & \cdots \\
0 & 0 & q & 0 & 1-q \\
& & \cdots & &
\end{array}\right)
$$


To evaluate the probabilities of the first return to the origin, we apply Theorem 2 , p. 86 , in [2]:

$$
\begin{array}{ll}
g_{1}^{1}=p, \quad g_{2 n}^{1}=\frac{1}{n}\left(\begin{array}{c}
2 n-2 \\
n-1
\end{array}\right) p^{n}(1-p)^{n}, \quad n \geq 1, \\
g_{1}^{2}=1, \quad g_{2 n}^{1}=\frac{1}{n}\left(\begin{array}{c}
2 n-2 \\
n-1
\end{array}\right) q^{n}(1-q)^{n}, \quad n \geq 1 .
\end{array}
$$

The moments of return to the origin are

$$
\begin{aligned}
& m_{0}^{1}=p+2 p(1-p) \sum_{n \geq 0}\left(\begin{array}{c}
2 n \\
n
\end{array}\right) p^{n}(1-p)^{n}, \\
& m_{0}^{2}=1+2 q(1-q) \sum_{n \geq 0}\left(\begin{array}{c}
2 n \\
n
\end{array}\right) q^{n}(1-q)^{n} .
\end{aligned}
$$

Now we evaluate the expectation of reaching the origin from some state $j>0$. For this, we count the number of trajectories leading from the state $j$ to 0 over the time $n$ that do not touch the origin. The total number of such trajectories equals the total number of trajectories from 0 to $j$ over time $n$ that do not touch the origin. By Theorem 1, p. 85, in 2] the latter number is equal to $\frac{j}{n}\left(\begin{array}{c}n \\ (n+j) / 2\end{array}\right)$. Then

$$
\begin{aligned}
& m_{j}^{1}=j \sum_{k \geq j}\left(\begin{array}{c}
k \\
(k+j) / 2
\end{array}\right) p^{(k+j) / 2}(1-p)^{(k-j) / 2}, \\
& m_{j}^{2}=j \sum_{k \geq j}\left(\begin{array}{c}
k \\
(k+j) / 2
\end{array}\right) q^{(k+j) / 2}(1-q)^{(k-j) / 2} .
\end{aligned}
$$

Finally, the expressions for the second moments of return to the origin are written as

$$
\begin{aligned}
& m_{0}^{1,2}=p+2 p(1-p) \sum_{n \geq 0}(n+1)\left(\begin{array}{c}
2 n \\
n
\end{array}\right) p^{n}(1-p)^{n}, \\
& m_{0}^{2,2}=1+2 q(1-q) \sum_{n \geq 0}(n+1)\left(\begin{array}{c}
2 n \\
n
\end{array}\right) q^{n}(1-q)^{n} .
\end{aligned}
$$

Theorem 5.3. Let $X$ and $X^{\prime}$ be two Markov chains with transient probabilities $P$ and $Q$, respectively, such that $p>1 / 2$ and $q>1 / 2$. Assume that the chains start from the states $i$ and $j$, respectively. Then the expectation of the coupling moment is finite. Moreover

$$
\mathrm{E}[T] \leq m_{i}^{1}+m_{j}^{1}+\gamma^{-1} \max \left\{\frac{m^{1,2}}{m_{0}^{1}}, \frac{m^{2,2}}{m_{0}^{2}}\right\} .
$$

As $\gamma$, one can choose $\min \left\{1 / m_{0}^{1}, 1 / m_{0}^{2}\right\}-\delta$ here where $\delta>0$ is arbitrary.

Proof. We are going to show that the condition $p>1 / 2$ yields the existence of the first two moments. Since

$$
\left(\begin{array}{c}
2 n \\
n
\end{array}\right) \sim \frac{4^{n}}{\sqrt{\pi n}}
$$

we have $4 p(1-p)<1$ and the series for $m_{0}^{1}$ is equivalent to the geometric progression and thus converges.

The finiteness of other moments is proved in the same fashion.

Therefore all expectations for both chains are finite. Aperiodicity follows from the inequality $g_{1}^{l}>0$.

As $\gamma$, one can choose $\min \left\{1 / m_{0}^{1}, 1 / m_{0}^{2}\right\}-\delta$ where $\delta>0$ is arbitrary. Then the renewal theorem implies that all $u_{n}^{l}$ are greater than $\gamma$ starting with some number. 


\section{Applications for inhomogeneous Markov Chains}

We consider inhomogeneous analogues of examples discussed in the preceding sections.

Inverse renewal chain. Consider two inhomogeneous Markov chains defined by the sets of matrices of transient probabilities at step $t$ :

$$
P_{t}=\left(\begin{array}{ccccc}
b_{0}^{(t)} & 1-b_{0}^{(t)} & 0 & 0 & \cdots \\
b_{1}^{(t)} & 0 & 1-b_{1}^{(t)} & 0 & \cdots \\
b_{2}^{(t)} & 0 & 0 & 1-b_{2}^{(t)} & \cdots \\
b_{3}^{(t)} & 0 & 0 & 0 & 1-b_{3}^{(t)}
\end{array}\right)
$$

and

$$
Q_{t}=\left(\begin{array}{ccccc}
c_{0}^{(t)} & 1-c_{0}^{(t)} & 0 & 0 & \ldots \\
c_{1}^{(t)} & 0 & 1-c_{1}^{(t)} & 0 & \ldots \\
c_{2}^{(t)} & 0 & 0 & 1-c_{2}^{(t)} & \ldots \\
c_{3}^{(t)} & 0 & 0 & 0 & 1-c_{3}^{(t)}
\end{array}\right) .
$$

Like the homogeneous case, the sequences $G_{n}^{t, l}$ and $g_{n}^{t, l}$ can be evaluated in an explicit form as follows:

$$
\begin{gathered}
G_{n}^{t, 1}=\prod_{k=0}^{n-1}\left(1-b_{k}^{(t+k)}\right), \quad G_{n}^{t, 2}=\prod_{k=0}^{n-1}\left(1-c_{k}^{(t+k)}\right), \quad n \geq 1, \\
g_{1}^{t, 1}=b_{0}^{(t)}, \quad g_{n}^{t, 1}=\prod_{k=0}^{n-2}\left(1-b_{k}^{(t+k)}\right) b_{n-1}^{t+n-1}, \quad n \geq 2, \\
g_{1}^{t, 1}=c_{0}^{(t)}, \quad g_{n}^{t, 2}=\prod_{k=0}^{n-2}\left(1-c_{k}^{(t+k)}\right) c_{n-1}^{(t+n-1)}, \quad n \geq 2 .
\end{gathered}
$$

The expectation $m_{j}^{l}$ of the time of reaching the origin from a state $j$ is given by

$$
m_{j}^{t, 1}=1+\sum_{k \geq 1} \prod_{i=0}^{k-1}\left(1-b_{j+i}^{(t+i)}\right), \quad m_{j}^{t, 2}=1+\sum_{k \geq 1} \prod_{i=0}^{k-1}\left(1-c_{j+i}^{(t+i)}\right) .
$$

Note that a bound for the expectation of the coupling moment is not known for the inhomogeneous case. In addition, such a result would require more complicated conditions as compared to those in Theorem 3.1. In the case under consideration, we derive the uniform integrability from the property that a sequence is uniformly integrable if the second moments are uniformly bounded.

The second moments of the return time to the origin are

$$
\begin{aligned}
& m^{1,2, t}=b_{0}^{(t)}+\sum_{n \geq 2} n^{2} \prod_{k=0}^{n-2}\left(1-b_{k}^{(t+k)}\right) b_{n-1}^{(t+n-1)}, \\
& m^{2,2, t}=c_{0}^{(t)}+\sum_{n \geq 2} n^{2} \prod_{k=0}^{n-2}\left(1-c_{k}^{(t+k)}\right) c_{n-1}^{(t+n-1)} .
\end{aligned}
$$

Now we are ready to state a result on the finiteness of the expectation of the coupling moment. 
Theorem 6.1. Let independent and time inhomogeneous Markov chains $X_{n}$ and $X_{n}^{\prime}$ whose transient probabilities at step $t$ are $P_{t}$ and $Q_{t}$ start from the initial states $l$ and $j$, respectively. Assume that

(1) $\inf _{t, n}\left\{b_{n}^{(t)}, c_{n}^{(t)}\right\}>0, n \geq 1$;

(2) $\sup \left\{m_{l}^{t, 1}, m_{j}^{t, 2}, m_{0}^{t, l}, m^{l, 2, t}\right\}<\infty$.

Then the expectation of the moment when the chains simultaneously visit the origin is finite; that is, $\mathrm{E}[T]<\infty$.

Proof. Condition (1) implies that the $g_{n}^{t, l}, n \geq 2$, are separated from zero. Thus condition (2) of Theorem 3.1 holds by Theorem 4.1. Condition (2) of Theorem 6.1 implies that the distributions $g_{n}^{t, l}$ are uniformly integrable.

Now Theorem 3.1 implies that the expectation of the first visit to zero is finite.

Remark 6.1. As above, we require neither $b_{0}^{(t)}>0$ nor $g_{1}^{t, l}>0$.

Random walk. Consider two inhomogeneous Markov chains defined by the following matrices of transient probabilities at step $t$ :

$$
P_{t}=\left(\begin{array}{ccccc}
p^{(t)} & 1-p^{(t)} & 0 & 0 & \cdots \\
p^{(t)} & 0 & 1-p^{(t)} & 0 & \cdots \\
0 & p^{(t)} & 0 & 1-p^{(t)} & \cdots \\
0 & 0 & p^{(t)} & 0 & 1-p^{(t)} \\
& & \cdots & &
\end{array}\right)
$$

and

$$
Q_{t}=\left(\begin{array}{ccccc}
q^{(t)} & 1-q^{(t)} & 0 & 0 & \cdots \\
q^{(t)} & 0 & 1-q^{(t)} & 0 & \cdots \\
0 & q^{(t)} & 0 & 1-q^{(t)} & \ldots \\
0 & 0 & q^{(t)} & 0 & 1-q^{(t)}
\end{array}\right)
$$

Note that the probability distribution of the renewal sequence cannot be evaluated explicitly in this case. Nevertheless it can be estimated effectively under certain assumptions. The following result explains the details.

Theorem 6.2. Let $X$ and $X^{\prime}$ be two time inhomogeneous Markov chains with the transient probabilities $P_{t}$ and $Q_{t}$ at step $t$. Assume that there are $p>1 / 2$ and $q>1 / 2$ such that $p^{(t)} \geq p$ and $q^{(t)} \geq q$. Let the chains start from the initial states $i$ and $j$, respectively. Then the expectation of the coupling moment is finite; that is,

$$
\mathrm{E}[T]<\infty .
$$

Proof. If $p^{(t)} \geq p>1 / 2$, then $p^{(t)}\left(1-p^{(t)}\right)<p(1-p)$, from which

$$
\begin{array}{cl}
g_{1}^{t, 1}=p^{(t)}, \quad & g_{2 n}^{t, 1} \leq \frac{1}{n}\left(\begin{array}{c}
2 n-2 \\
n-1
\end{array}\right) p^{n}(1-p)^{n}, \quad n \geq 1, \\
g_{1}^{t, 2}=1, \quad g_{2 n}^{t, 1} \leq \frac{1}{n}\left(\begin{array}{c}
2 n-2 \\
n-1
\end{array}\right) q^{n}(1-q)^{n}, \quad n \geq 1 .
\end{array}
$$

Now we estimate the expectations of the return times to the origin:

$$
\begin{aligned}
& m_{0}^{t, 1} \leq p+2 p(1-p) \sum_{n \geq 0}\left(\begin{array}{c}
2 n \\
n
\end{array}\right) p^{n}(1-p)^{n}, \\
& m_{0}^{t, 2} \leq 1+2 q(1-q) \sum_{n \geq 0}\left(\begin{array}{c}
2 n \\
n
\end{array}\right) q^{n}(1-q)^{n} .
\end{aligned}
$$


Next we evaluate the expectation of reaching the origin from a certain state $j>0$. For this, we count the total number of trajectories leading from the state $j$ to 0 over time $n$ that do not touch the origin. Note that the number of such trajectories equals the total number of trajectories leading from 0 to $j$ over time $n$ that do not touch the origin. By Theorem 1, p. 85, in 2] the later number is $\frac{j}{n}\left(\begin{array}{c}n \\ (n+j) / 2\end{array}\right)$. Thus

$$
\begin{aligned}
& m_{j}^{t, 1} \leq j \sum_{k \geq j}\left(\begin{array}{c}
k \\
(k+j) / 2
\end{array}\right) p^{(k+j) / 2}(1-p)^{(k-j) / 2} \\
& m_{j}^{t, 2} \leq j \sum_{k \geq j}\left(\begin{array}{c}
k \\
(k+j) / 2
\end{array}\right) q^{(k+j) / 2}(1-q)^{(k-j) / 2} .
\end{aligned}
$$

Finally we obtain the bounds for the second moments of return to the origin:

$$
\begin{aligned}
& m_{0}^{1,2, t}=p+2 p(1-p) \sum_{n \geq 0}(n+1)\left(\begin{array}{c}
2 n \\
n
\end{array}\right) p^{n}(1-p)^{n}, \\
& m_{0}^{2,2, t}=1+2 q(1-q) \sum_{n \geq 0}(n+1)\left(\begin{array}{c}
2 n \\
n
\end{array}\right) q^{n}(1-q)^{n} .
\end{aligned}
$$

Since

$$
\left(\begin{array}{c}
2 n \\
n
\end{array}\right) \sim \frac{4^{n}}{\sqrt{\pi n}}
$$

and $4 p(1-p)<1$, we obtain the uniform boundedness of the second moments, from which the uniform integrability follows.

The condition $p^{(t)} \geq p>1 / 2$ implies that $g_{1}^{t, 1}>1 / 2$, while condition $q^{(t)} \geq q>1$ yields $g_{1}^{t, 2}>1 / 2$. Now condition (2) of Theorem 3.1 follows from Corollary 4.2 .

Taking into account the uniform integrability proved above, we show that the expectation of the moment of the simultaneous visit to the origin is finite.

\section{Proof OF OTHER THEOREMS}

Proof of Theorem 4.1. Recall that if positive integer numbers $l_{1}, \ldots, l_{m}$ are coprime, then there exists a number $n_{1}$ such that every $n \geq n_{1}$ admit the representation

$$
n=\sum_{k=1}^{m} a_{k} l_{k}
$$

where the $a_{k}$ are some positive integer numbers. This means that

$$
u_{n}^{(t)} \geq \prod_{k=1}^{m}\left(g_{l_{k}}^{(t)}\right)^{a_{k}}>0
$$

for all $n \geq n_{1}$.

The assumption of the theorem implies that there exists an integer number $l$ such that $\inf _{t} g_{l}^{(t)}>0$. We assume without loss of generality that $l<n_{1}$.

Put $\gamma_{0}:=\inf _{t} \min \left\{u_{n}^{(t)}, n_{1}<n \leq n_{1}+l\right\}>0$. Then

$$
u_{n_{1}+l+1}^{(t)}=\sum_{n=0}^{n_{1}+l+1} g_{n}^{(t)} u_{n_{1}+l+1-n}^{(t+n)} \geq \sum_{n=0}^{l} g_{n}^{(t)} u_{n_{1}+l+1-n}^{(t+n)} \geq \gamma_{0}\left(1-G_{l}^{(t)}\right) \geq \gamma_{0}\left(1-\hat{G}_{l}\right) \text {. }
$$

Thus, for all $t>0$ and $n>n_{1}+l$,

$$
u_{n}^{(t)} \geq \gamma_{0} \prod_{n \geq l}\left(1-\hat{G}_{n}\right) .
$$



that

Consider the functional $F(G)=\prod_{n \geq l}\left(1-G_{l}\right)$ defined for sequences $G_{n}, n \geq l$, such

1) $G_{n}$ decreases to zero,

2) $0 \leq G_{n} \leq \hat{G}_{l}$,

3) $\sum_{n \geq l} G_{l}=\hat{\mu}-\hat{G}_{l-1}$.

This functional is concave, while the set of admissible points $G$ is convex. Thus the infimum of the functional $F(G)$ is attained at boundary points, from which

$$
\inf _{G} F(G)=\left(1-\hat{G}_{l}\right)^{\frac{\hat{\mu}-\hat{G}_{l}}{\hat{G}_{l}}} .
$$

Then one can choose $\gamma_{0}\left(1-\hat{G}_{l}\right)^{\frac{\hat{\mu}-\hat{G}_{l}}{\hat{G}_{l}}}$ as $\gamma$.

Proof of Theorem 4.2, We use induction starting with $2 n_{0}$ :

$$
u_{2 n_{0}}^{(k)} \geq\left(g_{n_{0}}^{(k)}\right)^{2}>\gamma_{0}^{2} .
$$

Assume that the statement holds for all $k$ and for all $j=2 n_{0}, \ldots, n$. We are going to show that it holds for $n+1$, too, where $n+1 \geq 3 n_{0}$. Indeed,

$$
\begin{aligned}
u_{n+1}^{(k)} & =\sum_{j=0}^{n+1} g_{j}^{(k)} u_{n+1-j}^{k+j} \geq \sum_{j=n_{0}}^{n+1} g_{j}^{(k)} u_{n+1-j}^{k+j} \\
& \geq \sum_{j=n_{0}}^{n+1-2 n_{0}} g_{j}^{(k)} u_{n+1-j}^{k+j} \geq \gamma^{(n)}\left(G_{n_{0}}^{(k)}-G_{n+1-2 n_{0}}^{(k)}\right) \\
& \geq \gamma^{(n)}\left(G_{n_{0}}^{(k)}-\hat{G}_{n+1-2 n_{0}}\right) \geq \gamma^{(n)}\left(a-\hat{G}_{n+1-2 n_{0}}\right),
\end{aligned}
$$

where

$$
a:=\inf _{k}\left(G_{n_{0}}^{(k)}\right) \geq \sum_{i=0}^{n_{0}-1} \gamma_{i}>0
$$

The solution is $\gamma^{(n+1)}=\gamma^{(n)}\left(a-\hat{G}_{n+1-2 n_{0}}\right)$.

Now we show that

$$
\prod_{i \geq n_{0}}\left(a-\hat{G}_{i}\right)>0 .
$$

Consider the functional

$$
F(G)=\prod_{i \geq 1}\left(a-G_{i}\right)
$$

defined for sequences $G_{i}$ such that

$$
0 \leq G_{i} \leq a-\gamma_{0}, \quad \sum_{i} G_{i} \leq \hat{m} .
$$

This functional is convex and thus attains its minimum at the boundary. In other words, the minimum is attained at a sequence such that

$$
\sum_{i} G_{i}=\hat{m}, \quad G_{i} \in\left\{0, a-\gamma_{0}\right\} .
$$

Hence $\hat{m} /\left(a-\gamma_{0}\right)$ elements equal $a-\gamma_{0}$, while the rest of them are 0 .

Then

$$
F(G) \geq \gamma_{0}^{\hat{m} /\left(a-\gamma_{0}\right)}=\exp \left(\frac{\hat{m} \ln \gamma_{0}}{a-\gamma_{0}}\right)=\exp \left(\frac{\hat{m} \ln \gamma_{0}}{\sum_{i=1}^{n_{0}-1} \gamma_{i}}\right) .
$$


Proof of Corollary 4.2. If a sequence is dominated and $\hat{g}_{1}>0$, then $g_{1}^{(k)} \geq \hat{g}_{1}>0$ for all $k$, and thus all assumptions of Theorem 4.2 hold with $n_{0}=1$ and $\gamma_{0}=\hat{g}_{1}$.

Proof of Theorem 4.3. It is clear that every finite set of different distributions is dominated. Since each of the distributions is aperiodic, there exists $n_{0}$ such that $g_{n_{0}}^{(k)}>0$. At most $k$ distributions are different among $g_{n}^{(k)}$ and thus one can choose $\gamma_{i}=\min _{k} g_{n_{0}+i}^{(k)}$, $i=0, \ldots, n_{0}-1$.

Proof of Theorem 4.4. First we assume that $\theta_{0}^{2}=0$.

Note that

$$
\begin{gathered}
\mathrm{E}\left[D_{n}\right] \leq \mu \mathrm{E}\left[U_{n}\right]-n, \\
\mathrm{E}\left[U_{n}\right] \leq n / \mu+\mu_{2} /(\mu)^{2}
\end{gathered}
$$

for an arbitrary homogeneous renewal process where $U_{n}$ is the number of renewals over the time $n$ and $\mu$ is the mean renewal time (see [14, p. 26] or [25]).

Combining inequalities (11) and (12) we get

$$
\mathrm{E}\left[B_{n} \mid \mathfrak{B}_{n-1}\right] \leq C:=\max \left\{\frac{\mu_{2}^{(1)}}{\mu^{(1)}}, \frac{\mu_{2}^{(2)}}{\mu^{(2)}}\right\} .
$$

Therefore

$$
\begin{gathered}
T \leq \theta_{0}^{1}+\sum_{n=0}^{\tau} B_{n}=\theta_{0}^{1}+C \sum_{n \geq 0} \mathbb{1}_{\tau \geq n}, \\
\mathrm{E}[T]=\mathrm{E}\left[\theta_{0}^{1}\right]+C \mathrm{E}[\tau] \leq \mathrm{E}\left[\theta_{0}^{1}\right]+C / \gamma_{0} \mathrm{E}\left[\theta_{0}^{1}\right]+\gamma^{-1} \max \left\{\frac{\mu_{2}^{(1)}}{\mu^{(1)}}, \frac{\mu_{2}^{(2)}}{\mu^{(2)}}\right\} .
\end{gathered}
$$

In the general case where the equality $\theta_{0}^{2}=0$ is not necessarily true,

$$
\mathrm{E}[T] \leq \max \left\{\mathrm{E}\left[\theta_{0}^{1}\right], \mathrm{E}\left[\theta_{0}^{2}\right]\right\}+\gamma^{-1} \max \left\{\frac{\mu_{2}^{(1)}}{\mu^{(1)}}, \frac{\mu_{2}^{(2)}}{\mu^{(2)}}\right\} .
$$

\section{BIBLIOGRAPHY}

1. W. Doeblin, Expose de la theorie des chaines simples constantes de Markov a un nomber fini d'estats, Mathematique de l'Union Interbalkanique 2 (1938), 77-105.

2. W. Feller, An Introduction to Probability Theory and its Applications, vol. 1, John Wiley \& Sons, New York, 1966. MR0228020 (37 \#3604)

3. N. V. Kartashov, Strong Stable Markov Chains, VSP, Utrecht/TViMS Scientific Publishers, Kiev, 1996. MR1451375 (99e:60150)

4. N. V. Kartashov, Exponential asymptotics of matrices of the Markov renewal, Asymptotic Problems for Stochastic Processes, Preprint 77-24, Institute of Mathematics of Academy of Science of Ukraine, Kiev, 1977, pp. 2-43. (Russian)

5. E. Nummelin, A splitting technique for Harris recurrent chains, Z. Wahrscheinlichkeitstheorie Verw. Geb. 43 (1978), 309-318. MR0501353 (58:18732)

6. E. Nummelin and R. L. Tweedie, Geometric ergodicity and R-positivity for general Markov chains, Ann. Probab. 6 (1978), 404-420. MR0474504 (57:14143)

7. T. Lindvall, On coupling of discrete renewal sequences, Z. Wahrscheinlichkeitstheorie Verw. Geb. 48 (1979), 57-70. MR.533006 (80g:60091)

8. I. N. Kovalenko and N. Ju. Kuznecov, Postroenie vlozhennogo protsessa vosstanovleniya dlya sushchestvenno mnogomernykh protsessov teorii massovogo obsluzhivaniya $i$ ego primenenie $k$ polucheniyu predelnykh teorem, Preprint 80, vol. 12, no. 80-12, Akad. Nauk Ukrain. SSR, Inst. Kibernet., Kiev, 1980. (Russian) MR612478(82i:60142)

9. P. Ney, A refinement of the coupling method in renewal theory, Stoch. Process. Appl. 11 (1981), 11-26. MR608004 (82d:60169) 
10. E. Numemelin and P. Tuominen, Geometric ergodicity of Harris recurrent Markov chains with applications to renewal theory, Stoch. Process. Appl. 12 (1982), 187-202. MR651903 (83f:60089)

11. E. Nummelin, General Irreducible Markov Chains and Nonnegative Operators, Cambridge University Press, Cambridge, 1984. MR776608 (87a:60074)

12. V. M. Zolotarev, Modern Theory of Summation of Independent Random Variables, "Nauka", Moscow, 1986; English transl., VSP, Utrecht, the Netherlands-Tokyo, Japan, 1997. MR1640024 (99m:60002)

13. S. T. Rachev, The Monge-Kantorovich problem on mass transfer and its applications in stochastics, Teor. Veroyatnost. i Primenen. 29 (1984), no. 4, 625-653. (Russian) MR773434 (86m:60026)

14. T. Lindvall, Lectures on the Coupling Method, John Wiley and Sons, $1991 . \quad$ MR.1180522 (94c:60002)

15. S. P. Mayn and R. L. Tweedie, Markov Chains and Stochastic Stability, Springer-Verlag, 1993. MR 1287609 (95j:60103)

16. P. Tuominen and R. Tweedie, Subgeometric rates of convergence of $f$-ergodic Markov chains, Adv. Appl. Probab. 26 (1994), 775-798. MR1285459 (95m:60097)

17. P. Tuominen and R. L. Tweedie, Subgeometric rates of convergence of f-ergodic Markov Chains, Adv. Appl. Probab. 26 (1994), 775-798. MR1285459 (95m:60097)

18. R. L. Tweedie and J. N. Corcoran, Perfect sampling of ergodic Harris chains, Ann. Appl. Probab. 11 (2001), no. 2, 438-451. MR1843053 (2002g:60111)

19. H. Thorisson, Coupling, Stationarity, and Regeneration, Springer, New York, 2000. MR 1741181 (2001b:60003)

20. S. F. Jarner and G. O. Roberts, Polynomial convergence rates of Markov chains, Ann. Appl. Probab. 12 (2001), 224-247. MR.1890063 (2003c:60117)

21. R. Douc, E. Moulines, and J. S. Rosenthal, Quantitative bounds for geometric convergence rates of Markov chains, Ann. Appl. Probab. 14 (2004), 1643-1664. MR2099647 (2005i:60146)

22. R. Douc, E. Moulines, and J. S. Rosenthal, Quantitative bounds on convergence of timeinhomogeneous Markov chains, Ann. Appl. Probab. 14 (2004), no. 4, 1643-1665. MR2099647 (2005i:60146)

23. R. Douc, E. Moulines, and P. Soulier, Practical drift conditions for subgeometric rates of convergence, Ann. Appl. Probab. 14 (2004), no. 4, 1353-1377. MR2071426 (2005e:60156)

24. R. Douc, E. Moulines, and P. Soulier, Computable convergence rates for subgeometrically ergodic Markov chains, Bernoulli 13 (2007), no. 3, 831-848. MR2348753(2008j:60172)

25. D. J. Daley, Tight bounds for the renewal function of a random walk, Ann. Probab. 8 (1980), no. 3, 615-621. MR573298 (81e:60094)

26. R. Douc, G. Fort, and A. Guillin, Subgeometric rates of convergence of $f$-ergodic strong Markov processes, Stoch. Process. Appl. 119 (2009), no. 3, 897-923. MR2499863 (2010j:60184)

27. V. V. Golomozyŭ and M. V. Kartashov, On integrability of the coupling moment for timeinhomogeneous Markov chains, Teor. Imovir. Matem. Statist. 89 (2014), 1-12; English transl. in Theor. Probability and Math. Statist. 89 (2014). MR 3235170

28. V. V. Golomozyı̆, Stability of non-homogeneous Markov chains, Visnyk Kyiv Univ., Ser. Fiz. Mat. Nauk 4 (2009), 10-15. (Ukrainian)

29. V. V. Golomozyı̆, A subgeometric estimate of the stability for time-homogeneous Markov chains, Teor. Imovir. Matem. Statist. 81 (2010), 31-46; English transl. in Theor. Probability and Math. Statist. 81 (2010), 35-50. MR.2667308 (2011c:60232)

30. M. V. Kartashov, Boundedness, limits, and stability of solutions of a perturbation of a nonhomogeneous renewal equation on a semiaxis, Teor. Imovir. Matem. Statist. 81 (2009), 6575; English transl. in Theor. Probability and Math. Statist. 81 (2010), 71-83. MR2667311 (2011f:60154)

31. M. V. Kartashov and V. V. Golomozyŭ, The mean coupling time for independent discrete renewal processes, Teor. Imovir. Matem. Statist. 84 (2011), 78-85; English transl. in Theor. Probability and Math. Statist. 84 (2012), 79-86. MR2857418 (2012f:60306)

32. M. V. Kartashov and V. V. Golomozy̆ , Maximal coupling procedure and stability of discrete Markov chains. I, Teor. Imovir. Matem. Statist. 86 (2012), 81-92; English transl. in Theor. Probability and Math. Statist. 86 (2013), 93-104. MR2986452

33. M. V. Kartashov and V. V. Golomozyı̆, Maximal coupling procedure and stability of discrete Markov chains. II, Teor. Imovir. Matem. Statist. 87 (2012), 58-70; English transl. in Theor. Probability and Math. Statist. 87 (2013), 65-78. MR3241447 
Department of Probability Theory, Statistics, and Actuarial Mathematics, Faculty for Mechanics and Mathematics, National Taras Shevchenko University, Volodymyrs'ka Street, 64, Kyiv 01601, UKRAINE

E-mail address: mailtower@gmail.com

Received 01/SEP/2013

Translated by N. SEMENOV 